\title{
Approximate Solution of Tuberculosis Disease Population Dynamics Model
}

\author{
Abdon Atangana ${ }^{1}$ and Necdet Bildik ${ }^{2}$ \\ ${ }^{1}$ Institute for Groundwater Studies, University of the Free State, P.O. Box 9300, Bloemfontein, South Africa \\ ${ }^{2}$ Department of Mathematics, Faculty of Art \& Sciences, Celal Bayar University, Muradiye Campus, 45047 Manisa, Turkey
}

Correspondence should be addressed to Abdon Atangana; abdonatangana@yahoo.fr

Received 22 March 2013; Accepted 2 June 2013

Academic Editor: R. K. Bera

Copyright (c) 2013 A. Atangana and N. Bildik. This is an open access article distributed under the Creative Commons Attribution License, which permits unrestricted use, distribution, and reproduction in any medium, provided the original work is properly cited.

We examine possible approximate solutions of both integer and noninteger systems of nonlinear differential equations describing tuberculosis disease population dynamics. The approximate solutions are obtained via the relatively new analytical technique, the homotopy decomposition method (HDM). The technique is described and illustrated with numerical example. The numerical simulations show that the approximate solutions are continuous functions of the noninteger-order derivative. The technique used for solving these problems is friendly, very easy, and less time consuming.

\section{Introduction}

Tuberculosis, MTB, or TB (short for tubercle bacillus) is a common and in many cases lethal, infectious disease caused by various strains of Mycobacterium, usually Mycobacterium Tuberculosis [1]. Tuberculosis typically attacks the lungs, but can also affect other parts of the body. It is spread through the air when people who have an active TB infection cough, sneeze, or otherwise transmit their saliva through the air [2]. Most infections are asymptomatic and latent, but about one in ten latent infections eventually progresses to active disease which, if left untreated, kills more than $50 \%$ of those so infected. Interested reader can find more about this model in [3-7].

Based on the standard SIRS model, the model population was compartmentalised into the susceptible $(S)$ and the infected $(I)$ which is further broken down into latently infected $\left(I_{L}\right)$ and actively infected $\left(I_{A}\right)$ while the recovered subpopulation is ploughed back into the susceptible group due to the possibility of reinfection after successful treatment of the earlier infection. The model monitors the temporary dynamics in the population of susceptible people $(t)$, TB latently infected people $I_{L}(t)$, and TB actively infected people
$I_{A}(t)$ as captured in the model system of ordinary differential equations that follows.

$$
\begin{aligned}
& \frac{d S(t)}{d t}=v f N-\alpha I_{A} S(t)+\delta S(t)+T_{A} I_{A}(t)+T_{L} I_{L}(t), \\
& \frac{d I_{L}(t)}{d t}=(1-P) \alpha I_{A} S(t)-\beta_{A} I_{L}(t)-T_{L} I_{L}(t)-\delta I_{L}(t), \\
& \frac{d I_{A}(t)}{d t}=P \alpha I_{A} S(t)+\beta_{A} I_{L}(t)-T_{A} I_{A}(t)-\delta I_{A}(t)-\varepsilon I_{A}(t)
\end{aligned}
$$

subject to the initial conditions

$$
S(0)=N, \quad I_{L}(0) \geq 0, \quad I_{A}(0) \geq 0,
$$

where $N$ is the total number of new people in the location of interest; $S$ is the number of susceptible people in the location; $I_{L}$ is the number of TB latently infected people; $I_{A}$ is the number of TB actively infected people; $v$ is the probability that a susceptible person is not vaccinated; $f$ is the efficient rate of vaccines; $T_{L}$ is the success rate of latent $T_{B}$ therapy; $T_{A}$ is the active TB treatment cure rate; $\alpha$ is the TB instantaneous 
incidence rate per susceptible; $\delta$ is humans natural death rate; $P$ is the proportion of infection instantaneously degenerating into active TB; $\varepsilon$ is the TB-induced death rate; and $\beta_{A}$ is the breakdown rate from latent to active TB. The equilibrium analysis of the model was studied in [8]. Equation (1) together with (2) does not have an exact solution and is usually solved numerically.

The purpose of this paper is to derive approximate analytical solutions for the standard form as well as the fractional version of (1) together with (2) using the relatively new analytical technique, the homotopy decomposition method (HDM).

The paper is structured as follows. In Section 2, we present the basic ideal of the homotopy decomposition method for solving partial differential equations. We present the application of the HDM for system Tuberculosis disease population dynamics model in Section 3. In Section 4, we present the application of the HDM for system of fractional Tuberculosis disease population dynamics model. The conclusions are then given finally in Section 5.

\section{Fundamental Information about Homotopy Decomposition Method}

To demonstrate the elementary notion of this technique, we consider a universal nonlinear nonhomogeneous partial differential equation with initial conditions of the following form [9-13].

$$
\begin{array}{r}
\frac{\partial^{m} U(x, t)}{\partial t^{m}}=L(U(x, t))+N(U(x, t))+f(x, t), \\
m=1,2,3 \ldots,
\end{array}
$$

focused on the primary condition

$$
\begin{array}{r}
\frac{\partial^{i} U(x, 0)}{\partial t^{i}}=y_{i}(x), \quad \frac{\partial^{m-1} U(x, 0)}{\partial t^{m-1}}=0, \\
i=0,1,2, \ldots, m-2,
\end{array}
$$

where $m$ is the order of the derivative, where $f$ is an identified function, $N$ is the common nonlinear differential operator, $L$ denotes a linear differential operator, and $m$ is the order of the derivative. The procedures first stage here is to apply the inverse operator $\partial^{m} / \partial t^{m}$ on both sides of (3) to obtain

$$
\begin{aligned}
& U(x, t)= \sum_{k=0}^{m-1} \frac{t^{k}}{k !} \frac{d^{k} u(x, 0)}{d t^{k}} \\
&+\int_{0}^{t} \int_{0}^{t_{1}} \cdots \int_{0}^{t_{m-1}} L(U(x, \tau)) \\
& \\
& \quad+N(U(x, \tau))+f(x, \tau) d \tau \cdots d t .
\end{aligned}
$$

The multi-integral in (3) can be transformed to

$$
\begin{aligned}
& \int_{0}^{t} \int_{0}^{t_{1}} \cdots \int_{0}^{t_{m-1}} L(U(x, \tau)) \\
& \quad+N(U(x, \tau))+f(x, \tau) d \tau \cdots d t \\
& =\frac{1}{(m-1) !} \int_{0}^{t}(t-\tau)^{m-1} L(U(x, \tau)) \\
& \quad+N(U(x, \tau))+f(x, \tau) d \tau
\end{aligned}
$$

so that (3) can be reformulated as

$$
\begin{aligned}
& U(x, t)=\sum_{k=0}^{m-1} \frac{t^{k}}{k !} y_{i}(x) \\
&+\frac{1}{(m-1) !} \int_{0}^{t}(t-\tau)^{m-1} L(U(x, \tau)) \\
& \quad+N(U(x, \tau))+f(x, \tau) d \tau .
\end{aligned}
$$

Using the homotopy scheme, the solution of the aforementioned integral equation is given in series form as

$$
\begin{gathered}
U(x, t, p)=\sum_{n=0}^{\infty} p^{n} U_{n}(x, t), \\
U(x, t)=\lim _{p \rightarrow 1} U(x, t, p),
\end{gathered}
$$

and the nonlinear term can be decomposed as

$$
N U(r, t)=\sum_{n=1}^{\infty} p^{n} \mathscr{H}_{n}(U),
$$

where $p \in(0,1]$ is an implanting parameter. $\mathscr{H}_{n}(U)$ is the polynomials that can be engendered by

$$
\mathscr{H}_{n}\left(U_{0}, \ldots, U_{n}\right)=\frac{1}{n !} \frac{\partial^{n}}{\partial p^{n}}\left[N\left(\sum_{j=0}^{n} p^{j} U_{j}(x, t)\right)\right],
$$

$$
n=0,1,2 \ldots .
$$

The homotopy decomposition method is obtained by the combination of decomposition method with Abel integral and is given by

$$
\begin{aligned}
& \sum_{n=0}^{\infty} p^{n} U_{n}(x, t) \\
& =T(x, t)+p \frac{1}{(m-1) !} \\
& \quad \times \int_{0}^{t}(t-\tau)^{m-1}\left[f(x, \tau)+L\left(\sum_{n=0}^{\infty} p^{n} U_{n}(x, \tau)\right)\right. \\
& \left.\quad+\sum_{n=0}^{\infty} p^{n} \mathscr{H}_{n}(U)\right] d \tau
\end{aligned}
$$

with

$$
T(x, t)=\sum_{k=0}^{m-1} \frac{t^{k}}{k !} y_{i}(x)
$$


Relating the terms of same powers of $p$, this gives solutions of various orders. The initial guess of the approximation is $T(x, t)$ that is actually the Taylor series of the exact solution of order $m$. Note that this initial guess insures the uniqueness of the series decompositions [9].

\section{Application of the HDM to the Model with Integer-Order Derivative}

In this section, we employ this method for deriving the set of the mathematical equations describing the tuberculosis disease population dynamics model.

Resulting from the steps involved in the HDM method, we reach at the following integral equations that are very simple to solve:

$$
\begin{aligned}
& p^{0}: S_{0}(t)=S(0) \\
& p^{0}: I_{L 0}(t)=I_{L}(0) \text {, } \\
& p^{0}: I_{A 0}(t)=I_{A}(0) \text {, } \\
& p^{1}: S_{1}(t) \\
& =\int_{0}^{t}\left(v f N-\alpha I_{A 0} S_{0}(\tau)+\delta S_{0}(\tau)\right. \\
& \left.+T_{A} I_{A 0}(\tau)+T_{L} I_{L 0}(\tau)\right) d \tau, \quad S_{1}(0)=0, \\
& p^{1}: I_{L 1}(t) \\
& =\int_{0}^{t}\left((1-P) \alpha I_{A 0} S_{0}(\tau)-\beta_{A} I_{L 0}(\tau)\right. \\
& \left.-T_{L} I_{L} 0(\tau)-\delta I_{L 0}(\tau)\right) d \tau, \quad I_{L 1}(0)=0, \\
& p^{1}: I_{A 1}(t) \\
& =\int_{0}^{t}\left(P \alpha I_{A 0} S_{0}(\tau)+\beta_{A} I_{L 0}(\tau)\right. \\
& \left.-T_{A} I_{A 0}(\tau)-\delta I_{A 0}(\tau)-\varepsilon I_{A 0}(\tau)\right) d \tau, \quad I_{A 1}(0)=0, \\
& p^{n}: S_{n}(t) \\
& =\int_{0}^{t}\left(v f N-\alpha \sum_{j=0}^{n-1} I_{A j} S_{n-j-1}(\tau)+\delta S_{n-1}(\tau)\right. \\
& \left.+T_{A} I_{A(n-1)}(\tau)+T_{L} I_{L(n-1)}(\tau)\right) d \tau, \\
& S_{n-1}(0)=0 \text {, } \\
& p^{n}: I_{L n}(t) \\
& =\int_{0}^{t}\left((1-P) \alpha \sum_{j=0}^{n-1} I_{A j} S_{n-j-1}(\tau)-\beta_{A} I_{L(n-1)}(\tau)\right.
\end{aligned}
$$

$$
\begin{array}{r}
\left.-T_{L} I_{L(n-1)}(\tau)-\delta I_{L(n-1)}(\tau)\right) d \tau \\
I_{L n}(0)=0
\end{array}
$$

$$
\begin{aligned}
& p^{n}: I_{A n}(t) \\
&=\int_{0}^{t}\left(P \alpha \sum_{j=0}^{n-1} I_{A j} S_{n-j-1}(\tau)+\beta_{A} I_{L(n-1)}(\tau)-T_{A} I_{A(n-1)}(\tau)\right. \\
&\left.\quad-\delta I_{A(n-1)}(\tau)-\varepsilon I_{A(n-1)}(\tau)\right) d \tau, \quad I_{A n}(0)=0 .
\end{aligned}
$$

Integrating the previous, we obtain the following components:

$$
\begin{gathered}
S_{0}(t)=S(0) ; \quad I_{L 0}(t)=I_{L}(0) ; \\
I_{A 0}(t)=I_{A}(0), \\
S_{1}(t)=\left(v f N-\alpha I_{A 0} S_{0}+\delta S_{0}+T_{A} I_{A 0}+T_{L} I_{L 0}\right) t, \\
I_{L 1}(t)=\left((1-P) \alpha I_{A 0} S_{0}-\beta_{A} I_{L 0}-T_{L} I_{L} 0-\delta I_{L 0}\right) t, \\
I_{A 1}(t)=\left(P \alpha I_{A 0} S_{0}+\beta_{A} I_{L 0}-T_{A} I_{A 0}-\delta I_{A 0}-\varepsilon I_{A 0}\right) t .
\end{gathered}
$$

For simplicity, let us put

$$
\begin{gathered}
a=\left(v f N-\alpha I_{A 0} S_{0}+\delta S_{0}+T_{A} I_{A 0}+T_{L} I_{L 0}\right), \\
b=\left((1-P) \alpha I_{A 0} S_{0}-\beta_{A} I_{L 0}-T_{L} I_{L} 0-\delta I_{L 0}\right), \\
c=\left(P \alpha I_{A 0} S_{0}+\beta_{A} I_{L 0}-T_{A} I_{A 0}-\delta I_{A 0}-\varepsilon I_{A 0}\right), \\
S_{2}(t)=\frac{1}{2} t^{2}\left(b T_{A}+c T_{L}-a I_{A 0} \alpha-b S_{0} \alpha+a \delta\right) \\
=\frac{t^{2}}{2} a_{1}, \quad \begin{array}{c}
2 \\
I_{L 2}(t)=\frac{1}{2} t^{2}\left(-c T_{L}+a I_{A 0} \alpha-a I_{A 0} P \alpha\right. \\
\left.+b S_{0} \alpha-c \beta_{A}-c \delta\right)=\frac{t^{2}}{2} b_{1}, \\
\left.+c \beta_{A}-b \delta-b \varepsilon\right)=c_{1} \frac{t^{2}}{2} .
\end{array} \\
I_{A 2}(t)=\frac{1}{2} t^{2}\left(a I_{A 0} P \alpha+b P \alpha S_{0}-b T_{A}\right.
\end{gathered}
$$

In general, we obtain the following recursive formulas:

$$
\begin{aligned}
& S_{n}(t)=\frac{t^{n}}{n !} a_{n}, \\
& I_{L n}(t)=\frac{t^{n}}{n !} b_{n}, \\
& I_{A n}(t)=c_{n} \frac{t^{n}}{n !},
\end{aligned}
$$


where $a_{n}, b_{n}$, and $c_{n}$ depend on the fixed set of empirical parameters. It therefore follows that the approximate solution of the system (1) is given as

$$
\begin{aligned}
& S_{N}(t)=\sum_{n=0}^{N} \frac{t^{n}}{n !} a_{n}, \\
& I_{L N}(t)=\sum_{n=0}^{N} \frac{t^{n}}{n !} b_{n}, \\
& I_{A N}(t)=\sum_{n=0}^{N} \frac{t^{n}}{n !} c_{n} .
\end{aligned}
$$

If for instance one supposes that the total number of new people in the location of interest is $N=100$; the initial number of susceptible people in the location is $S(0)=96$; the initial number of TB latently infected people is $I_{L}(0)=3$; the initial number of TB actively infected people is $I_{A}(0)=1$; the probability that a susceptible person is not vaccinated is $v=0.5$; the efficient rate of vaccines is $f=0.5$; the success rate of latent TB therapy is $T_{L}=0.8$; the active TB treatment cure rate is $T_{A}=0.74$; the TB instantaneous incidence rate per susceptible is $\alpha=0.41$; humans natural death rate is $\delta=1 /(366 \times 70)$; the proportion of infection instantaneously degenerating into active TB is $P=0.0197$; the TB-induced death rate is $\varepsilon=0.0735$; and the breakdown rate from latent to active TB is $\beta_{A}=0.01$, then the following approximate solution is obtained as a result of the first 8 terms of the series decomposition:

$$
\begin{aligned}
S(t)= & 96-11.2162 t+62.1069 t^{2}-29.5924 t^{3}-149.2 t^{4} \\
& +48.3455 t^{5}-20.6378 t^{6}+15.5857 t^{7}+\cdots \\
I_{L}(t)= & +36.8527 t-62.9161 t^{2}-797.302 t^{3}+151.174 t^{4} \\
& -48.8926 t^{5}+20.7629 t^{6}-15.6036 t^{7}+\cdots \\
I_{A}(t)= & 1-0.706394 t+0.252053 t^{2}-0.252832 t^{3} \\
& -1.96203 t^{4}+0.573666 t^{5}-0.131459 t^{6} \\
& +0.0190148 t^{7}+\cdots
\end{aligned}
$$

If in addition we assume that no new person migrates or is born in this area, we obtain the following figures. The approximate solutions of the main problem are depicted in Figures 1, 2, and 3, respectively.

Figure 1 shows that, if there is migration or newborn in the location of interest, the number of susceptible people will vanish as time goes, because of the natural death rate and due to TB. Note that any person that is latently infected is removed from the set of susceptible. Figure 2 indicates that the number of people that are latently infected will increase up to a certain time and then vanish as time goes. The number of susceptible people, will become latently infected since some are not vaccinated against the TB and finally will vanish due to. Figure 3 indicates that the number of TB actively infected

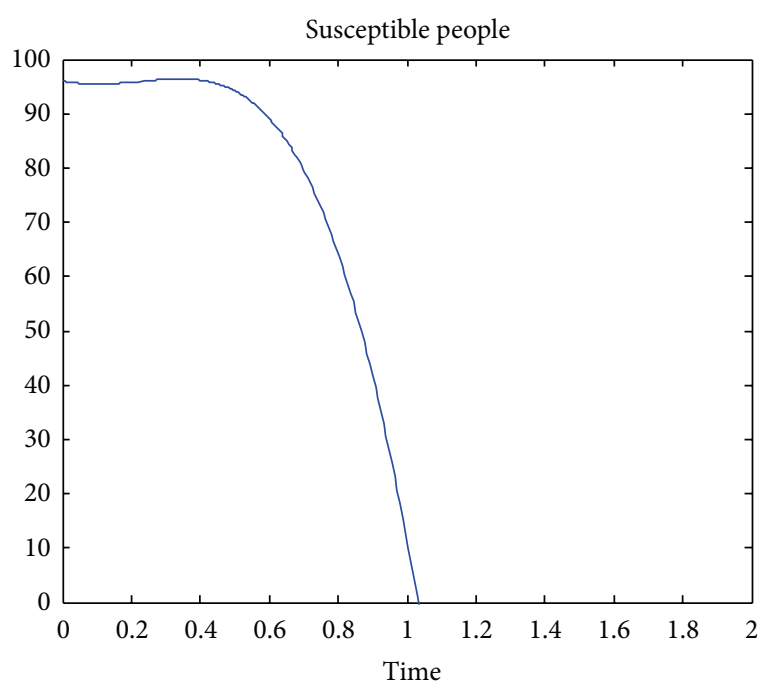

FIGURE 1: Approximate solution for the number of susceptible people in the location.

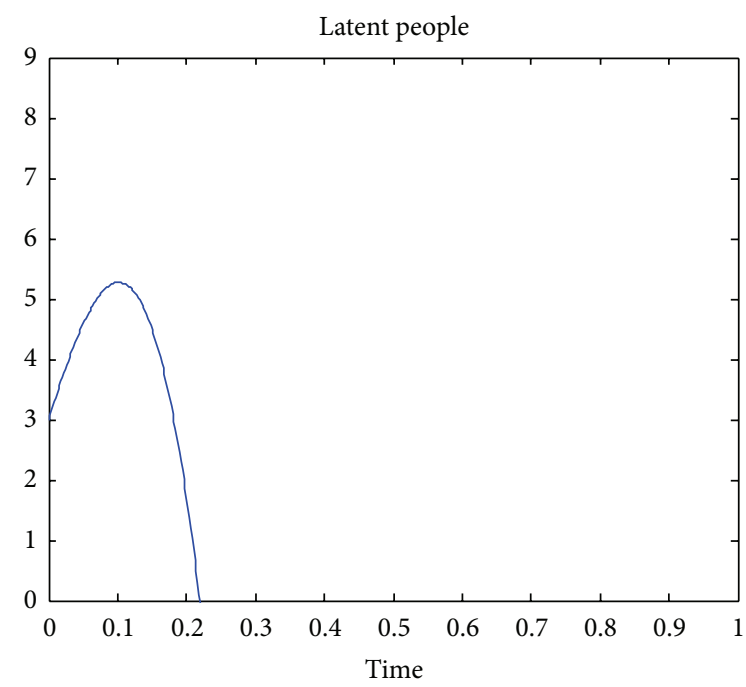

FIGURE 2: Approximate solution for the number of TB latently infected people.

people will also vanish because of the natural death rate and the death due to TB.

\section{Application of the HDM to the Model with Noninteger-Order Derivative}

Fractional calculus has been used to model physical and engineering processes, which are found to be best described by fractional differential equations. It is worth noting that the standard mathematical models of integer-order derivatives, including nonlinear models, do not work adequately in many cases. In the recent years, fractional calculus has played a very important role in various fields such as mechanics, electricity, chemistry, biology, economics, notably control theory, and signal and image processing. Major topics 


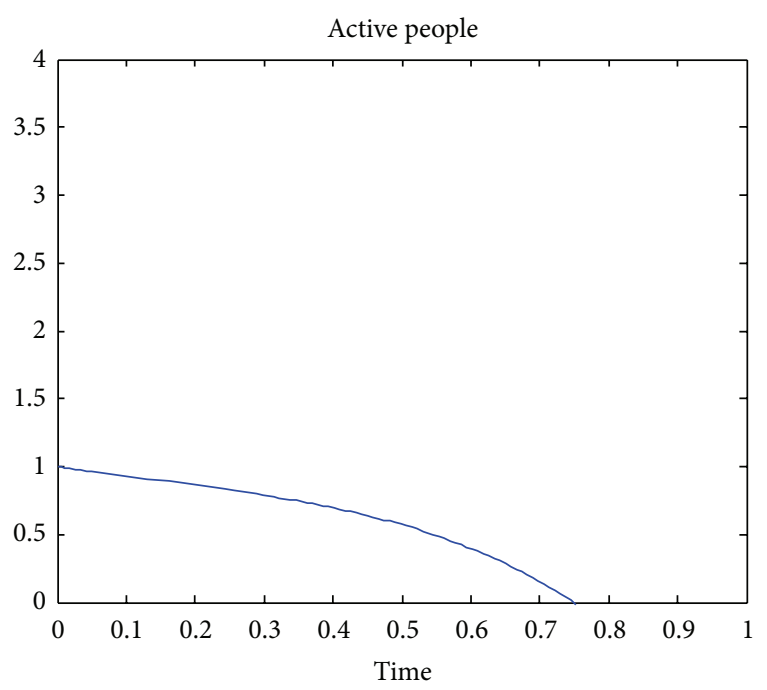

Figure 3: Approximate solution for the number of TB actively infected people.

include anomalous diffusion; vibration and control; continuous time random walk; Levy statistics, fractional Brownian motion; fractional neutron point kinetic model; power law; Riesz potential; fractional derivative and fractals; computational fractional derivative equations; nonlocal phenomena; history-dependent process; porous media; fractional filters; biomedical engineering; fractional phase-locked loops, and groundwater problem (see [14-21]).

\subsection{Properties and Definitions}

Definition 1. A real function $f(x), x>0$, is said to be in the space $C_{\mu}, \mu \in \mathbb{R}$, if there exists a real number $p>\mu$, such that $f(x)=x^{p} h(x)$, where $h(x) \in C[0, \infty)$, and it is said to be in space $C_{\mu}^{m}$ if $f^{(m)} \in C_{\mu}, m \in \mathbb{N}$.

Definition 2. The Riemann-Liouville fractional integral operator of order $\alpha \geq 0$, of a function $f \in C_{\mu}, \mu \geq-1$, is defined as

$$
\begin{gathered}
J^{\alpha} f(x)=\frac{1}{\Gamma(\alpha)} \int_{0}^{x}(x-t)^{\alpha-1} f(t) d t, \quad \alpha>0, x>0 \\
J^{0} f(x)=f(x) .
\end{gathered}
$$

Properties of the operator can be found in [14-16]. We mention only the following: for $f \in C_{\mu}, \mu \geq-1, \alpha, \beta \geq 0$, and $\gamma>-1$,

$$
\begin{gathered}
J^{\alpha} J^{\beta} f(x)=J^{\alpha+\beta} f(x), \\
J^{\alpha} J^{\beta} f(x)=J^{\beta} J^{\alpha} f(x) J^{\alpha} x^{\gamma}=\frac{\Gamma(\gamma+1)}{\Gamma(\alpha+\gamma+1)} x^{\alpha+\gamma} .
\end{gathered}
$$

Lemma 3. If $m-1<\alpha \leq m, m \in \mathbb{N}$, and $f \in C_{\mu}^{m}, \mu \geq-1$, then

$$
\begin{gathered}
D^{\alpha} J^{\alpha} f(x)=f(x) \\
J^{\alpha} D_{0}^{\alpha} f(x)=f(x)-\sum_{k=0}^{m-1} f^{(k)}\left(0^{+}\right) \frac{x^{k}}{k !}, \quad x>0 .
\end{gathered}
$$

Definition 4 (partial derivatives of fractional order). Assume now that $f(\mathbf{x})$ is a function of $n$ variables $x_{i} i=1, \ldots, n$ also of class $C$ on $D \in \mathbb{R}_{n}$. We define partial derivative of order $\alpha$ for $f$ respect to $x_{i}$ the function

$$
a \partial_{\underline{\mathbf{x}}}^{\alpha} f=\left.\frac{1}{\Gamma(m-\alpha)} \int_{a}^{x_{i}}\left(x_{i}-t\right)^{m-\alpha-1} \partial_{x_{i}}^{m} f\left(x_{j}\right)\right|_{x_{j}=t} d t .
$$

where $\partial_{x_{i}}^{m}$ is the usual partial derivative of integer-order $m$.

4.2. Approximate Solution of Fractional Version. The system of equations under investigation here is given as

$$
\begin{aligned}
\frac{d^{\mu} S(t)}{d t^{\mu}}= & v f N-\alpha I_{A} S(t)+\delta S(t) \\
& +T_{A} I_{A}(t)+T_{L} I_{L}(t), \quad 0<\mu \leq 1, \\
\frac{d^{\eta} I_{L}(t)}{d t^{\eta}}= & (1-P) \alpha I_{A} S(t)-\beta_{A} I_{L}(t) \\
& -T_{L} I_{L}(t)-\delta I_{L}(t), \quad 0<\eta \leq 1, \\
\frac{d^{v} I_{A}(t)}{d t^{v}=} & P \alpha I_{A} S(t)+\beta_{A} I_{L}(t)-T_{A} I_{A}(t) \\
& -\delta I_{A}(t)-\varepsilon I_{A}(t), \quad 0<v \leq 1 .
\end{aligned}
$$

Following the discussion presented earlier, we arrive at the following equations:

$$
\begin{gathered}
p^{0}: S_{0}(t)=S(0), \\
p^{0}: I_{L 0}(t)=I_{L}(0), \\
p^{0}: I_{A 0}(t)=I_{A}(0),
\end{gathered}
$$

$$
\begin{array}{r}
p^{1}: S_{1}(t) \\
=\frac{1}{\Gamma(\mu)} \int_{0}^{t}(t-\tau)^{\mu-1} \\
\times\left(v f N-\alpha I_{A 0} S_{0}(\tau)+\delta S_{0}(\tau)\right. \\
\left.+T_{A} I_{A 0}(\tau)+T_{L} I_{L 0}(\tau)\right) d \tau, \\
S_{1}(0)=0,
\end{array}
$$

$$
\begin{aligned}
& p^{1}: I_{L 1}(t) \\
&=\frac{1}{\Gamma(\eta)} \int_{0}^{t}(t-\tau)^{\eta-1} \\
& \times\left((1-P) \alpha I_{A 0} S_{0}(\tau)-\beta_{A} I_{L 0}(\tau)\right. \\
&\left.-T_{L} I_{L} 0(\tau)-\delta I_{L 0}(\tau)\right) d \tau, \\
& I_{L 1}(0)=0,
\end{aligned}
$$

$$
\begin{aligned}
& p^{1}: I_{A 1}(t) \\
& =\frac{1}{\Gamma(v)} \int_{0}^{t}(t-\tau)^{v-1} \\
& \quad \times\left(P \alpha I_{A 0} S_{0}(\tau)+\beta_{A} I_{L 0}(\tau)\right. \\
& \left.\quad-T_{A} I_{A 0}(\tau)-\delta I_{A 0}(\tau)-\varepsilon I_{A 0}(\tau)\right) d \tau, \\
& \quad I_{A 1}(0)=0,
\end{aligned}
$$




$$
\begin{aligned}
& p^{n}: S_{n}(t) \\
& =\frac{1}{\Gamma(\mu)} \int_{0}^{t}(t-\tau)^{\mu-1} \\
& \quad \times\left(v f N-\alpha \sum_{j=0}^{n-1} I_{A j} S_{n-j-1}(\tau)+\delta S_{n-1}(\tau)\right. \\
& \left.\quad+T_{A} I_{A(n-1)}(\tau)+T_{L} I_{L(n-1)}(\tau)\right) d \tau, \\
& \quad S_{n-1}(0)=0
\end{aligned}
$$

$$
\begin{aligned}
& p^{n}: I_{L n}(t) \\
& =\frac{1}{\Gamma(\eta)} \int_{0}^{t}(t-\tau)^{\eta-1} \\
& \times\left((1-P) \alpha \sum_{j=0}^{n-1} I_{A j} S_{n-j-1}(\tau)-\beta_{A} I_{L(n-1)}(\tau)\right. \\
& \left.-T_{L} I_{L(n-1)}(\tau)-\delta I_{L(n-1)}(\tau)\right) d \tau, \\
& I_{L n}(0)=0 \text {, }
\end{aligned}
$$

$$
\begin{aligned}
& p^{n}: I_{A n}(t) \\
&=\frac{1}{\Gamma(v)} \int_{0}^{t}(t-\tau)^{v-1} \\
& \times\left(\begin{array}{l}
P \alpha \sum_{j=0}^{n-1} I_{A j} S_{n-j-1}(\tau)+\beta_{A} I_{L(n-1)}(\tau) \\
-T_{A} I_{A(n-1)}(\tau)-\delta I_{A(n-1)}(\tau) \\
\left.-\varepsilon I_{A(n-1)}(\tau)\right)
\end{array}\right) d \tau \\
& I_{A n}(0)=0 .
\end{aligned}
$$

Integrating the previous, we obtain the following components:

$$
\begin{gathered}
S_{0}(t)=S(0) ; \quad I_{L 0}(t)=I_{L}(0) ; \\
I_{A 0}(t)=I_{A}(0), \\
S_{1}(t)=-\frac{11.2162 t^{\mu}}{\Gamma(1+\mu)} ; \\
I_{L 1}(t)=\frac{36.8527 t^{\eta}}{\Gamma(1+\eta)}, \\
I_{A 1}(t)=-\frac{0.706394 t^{v}}{\Gamma(1+v)}, \\
\quad t^{\mu}\left(\frac{29.4822 t^{\eta}}{\Gamma(1+\eta+\mu)}+\frac{4.59822 t^{\mu}}{\Gamma(1+2 \mu)}\right. \\
\left.+\frac{27.2809 t^{v}}{\Gamma(1+v+\mu)}\right),
\end{gathered}
$$

$$
\begin{gathered}
I_{L 2}(t)=-t^{\eta}\left(\frac{29.8522 t^{\eta}}{\Gamma(1+2 \eta)}+\frac{4.58965 t^{\mu}}{\Gamma(1+\eta+\mu)}\right. \\
\left.+\frac{27.7492 t^{v}}{\Gamma(1+v+\mu)}\right)
\end{gathered}
$$$$
I_{A 2}(t)=t^{v}\left(\frac{0.368527 t^{\eta}}{\Gamma(1+\eta+v)}-\frac{0.00901337 t^{\mu}}{\Gamma(1+v+\mu)}\right.
$$$$
\left.+\frac{0.520184 t^{v}}{\Gamma(1+2 v)}\right)
$$$$
S_{3}(t)=t^{\mu}\left(-\frac{3.24846 t^{\mu+v} \Gamma(1+\mu+v)}{\Gamma(1+\mu) \Gamma(1+v) \Gamma(1+2 \mu+v)}\right.
$$$$
-\frac{0.298522 t^{2 \eta}}{\Gamma(1+2 \eta+v)}-\frac{15.7583 t^{\eta+\mu}}{\Gamma(1+\eta+2 \mu)}
$$$$
-\frac{1.88509 t^{2 \mu}}{\Gamma(1+3 \mu)}-\frac{36.4319 t^{\eta+v}}{\Gamma(1+\mu+v+\eta)}
$$$$
\left.-\frac{10.836 t^{\mu+v}}{\Gamma(1+2 \mu+v)}-\frac{20.0895 t^{2 v}}{\Gamma(1+\mu+2 v)}\right),
$$$$
I_{L 3}(t)=t^{\eta}\left(-\frac{1148.5 t^{2 \eta}}{\Gamma(1+3 \eta)}-\frac{164.513 t^{\eta+\mu}}{\Gamma(1+2 \eta+\mu)}+\frac{1.88158 t^{2 \mu}}{\Gamma(1+\eta+2 \mu)}\right.
$$$$
-\frac{1067.59 t^{\eta}}{\Gamma(1+2 \eta+v)}+\frac{11.1633 t^{\mu+v}}{\Gamma(1+\eta+\mu+v)}
$$$$
\left.+\frac{3.2421 t^{\mu+v} \Gamma(1+\mu+v)}{\Gamma(1+\mu) \Gamma(1+v) \Gamma(1+\eta+\mu+v)}\right),
$$$$
I_{A 3}(t)=t^{v}\left(\frac{0.00636699 t^{\mu+v} \Gamma(1+\mu+v)}{\Gamma(1+\mu) \Gamma(1+v) \Gamma(1+2 v+\mu)}\right.
$$$$
-\frac{0.298522 t^{2 \eta}}{\Gamma(1+2 \eta+v)}-\frac{0.0222046 t^{\eta+\mu}}{\Gamma(1+\eta+2 \mu)}
$$$$
+\frac{0.00369513 t^{2 \mu}}{\Gamma(1+3 \mu)}-\frac{0.548873 t^{\eta+v}}{\Gamma(1+\mu+2 v)}
$$$$
\left.+\frac{0.0285603 t^{\mu+v}}{\Gamma(1+\mu+2 v)}-\frac{0.38306 t^{2 v}}{\Gamma(1+3 v)}\right) .
$$

The remaining terms can be obtained in the same manner. But here only few terms of the series solutions are considered, and the asymptotic solution is given as

$$
\begin{gathered}
S(t)=S_{0}(t)+S_{1}(t)+S_{2}(t)+S_{3}(t)+\cdots, \\
I_{L}(t)=I_{L 0}(t)+I_{L 1}(x, t)+I_{L 2}(x, t)+I_{L 3}(x, t)+\cdots, \\
I_{A}(t)=I_{A 0}(t)+I_{A 1}(x, t)+I_{A 2}(x, t)+I_{A 3}(x, t)+\cdots .
\end{gathered}
$$

The following figures show the simulated solutions for different values of the fractional order derivatives. The approximate 


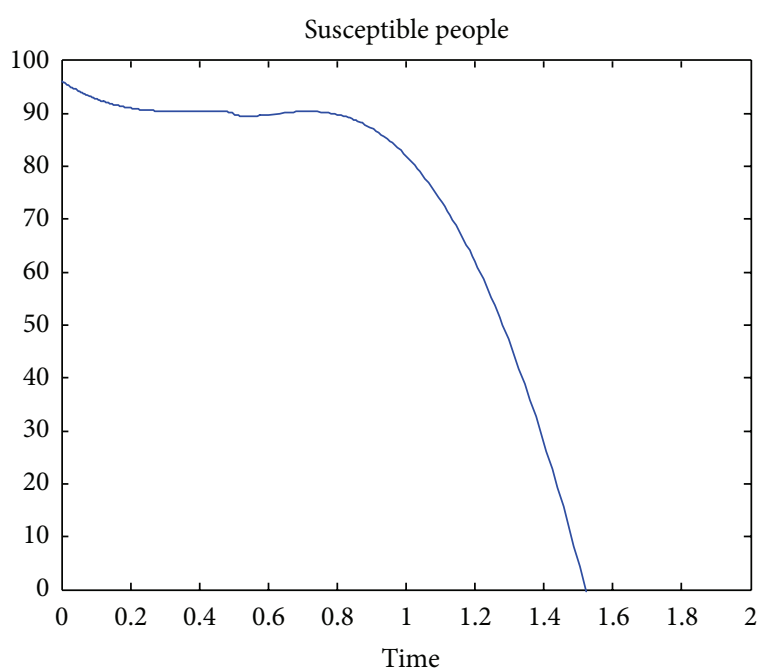

FIgURE 4: Approximate for $\mu=0.45, \eta=0.7$, and $v=0.85$.

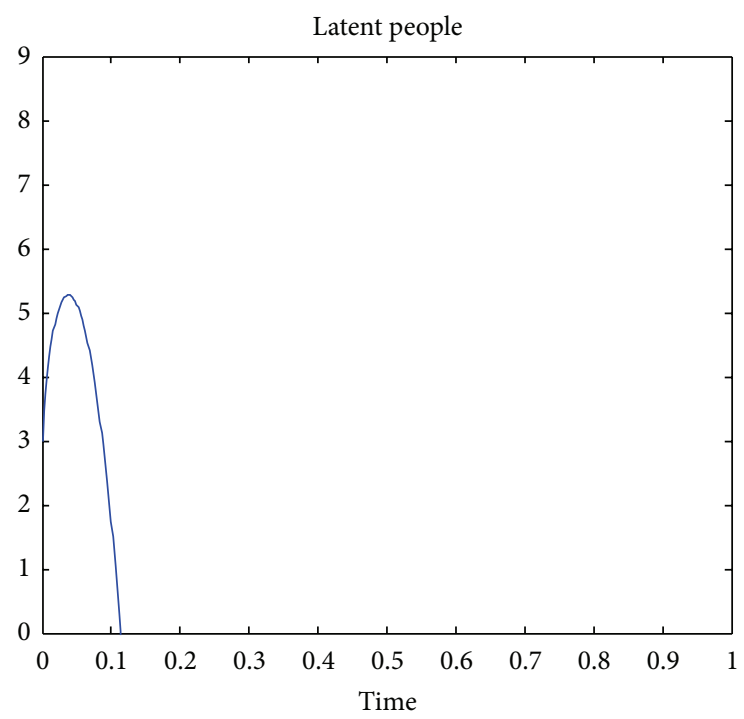

FIGURE 5: Approximate for $\mu=0.45, \eta=0.7$, and $v=0.85$.

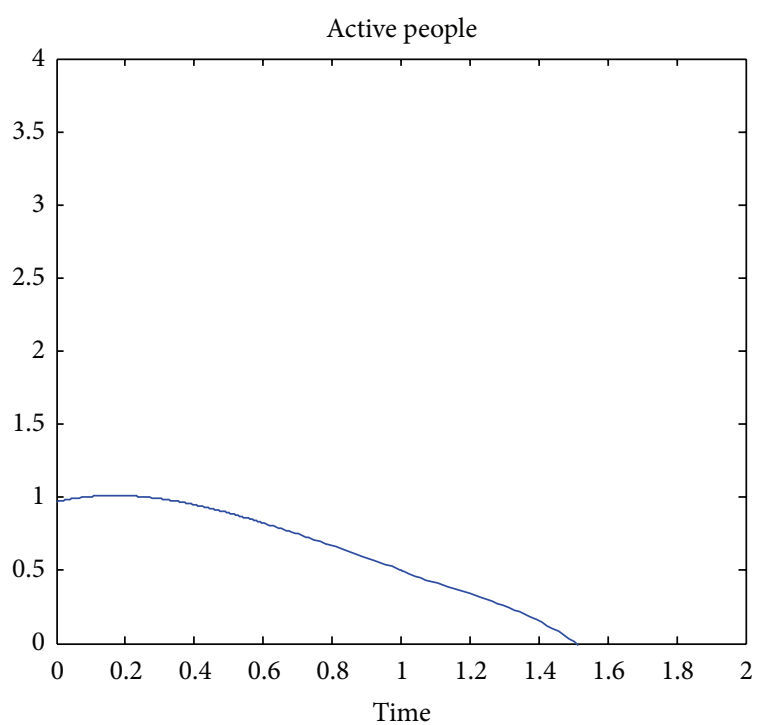

Figure 6: Approximate for $\mu=0.45, \eta=0.7$, and $v=0.85$.

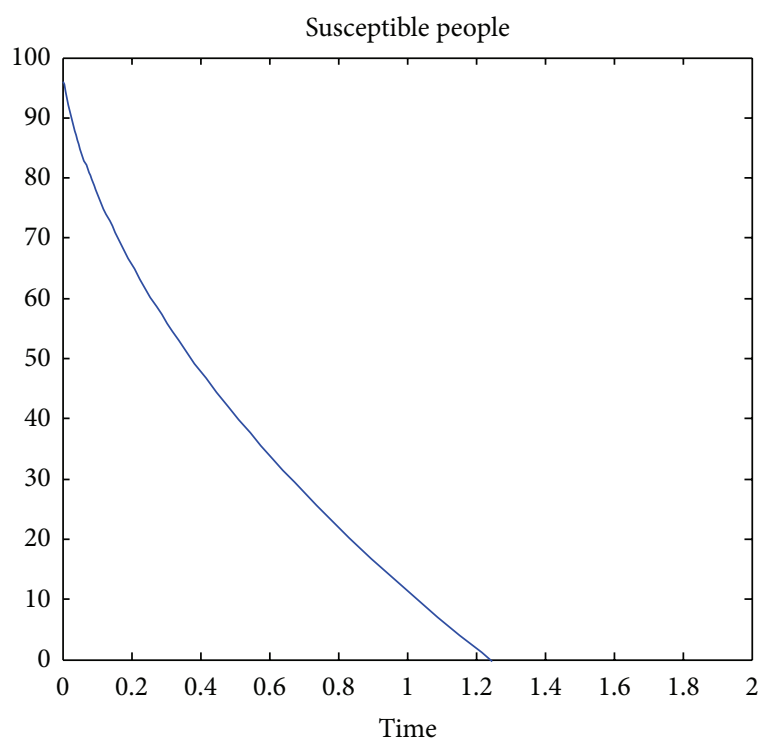

FIGURE 7: Approximate for $\mu=0.045, \eta=0.5$, and $v=0.085$.

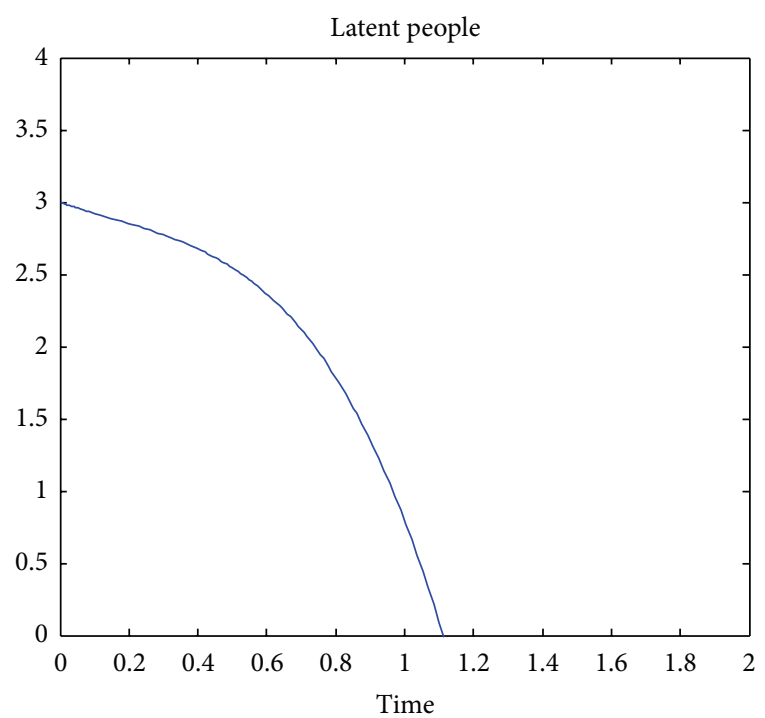

FIGURE 8: Approximate for $\mu=0.045, \eta=0.5$, and $v=0.085$.

solutions of the main problem are depicted in Figures 4, 5, 6, 7,8 , and 9 , respectively.

The numerical simulations show that the approximate solutions are continuous functions of the noninteger-order derivative. It is worth noting that the standard mathematical models of integer-order derivatives, including nonlinear models, do not work adequately in many cases. It is therefore advisable to use the fractional model for describing this problem.

\section{Conclusion}

The tuberculosis model was examined for the case of integerand noninteger-order derivatives. Both systems of nonlinear equations were solved with an iterative analytical model 


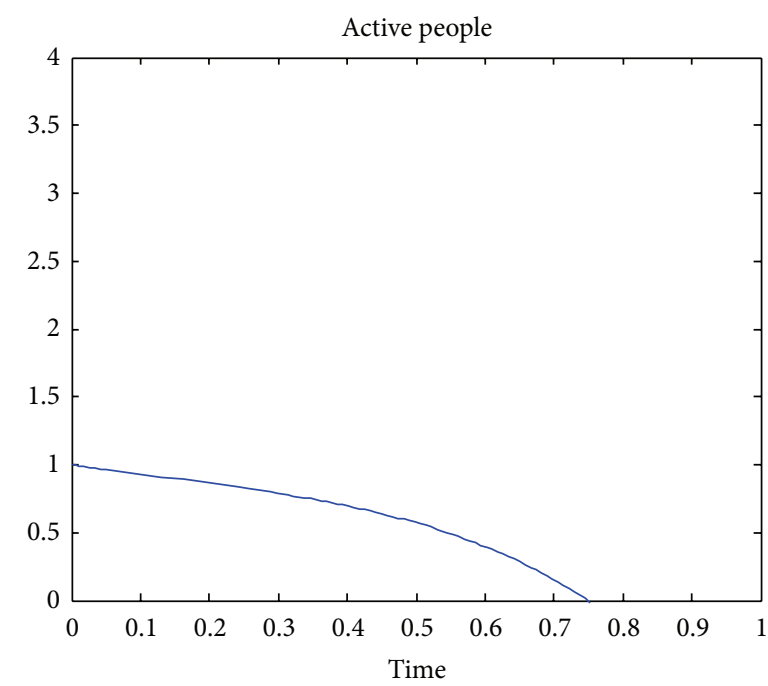

FiguRE 9: Approximate for $\mu=0.045, \eta=0.5$, and $v=0.085$.

called the homotopy decomposition model method. The basic characters of the relatively new technique are presented in detail. The approximate solutions of the noninteger case are increasing continuous functions of the fractional order derivative. The technique used for solving these problems is friendly, very easy, and less time consuming. The numerical solutions in both cases display the biological behaviour of the real world situation.

\section{References}

[1] V. Kumar, A. K. Abbas, N. Fausto, and R. N. Mitchell, Robbins Basic Pathology, Saunders Elsevier, 8th edition, 2007.

[2] A. Konstantinos, Testing For Tuberculosis, vol. 33, Australian Prescriber, 2010.

[3] K. G. Castro, "Global tuberculosis challenges," Emerging Infectious Disease, vol. 4, no. 3, pp. 408-409, 1998.

[4] C. Dye, Z. Fengzengb, S. Scheele et al., "Evaluating the impact of tuberculosis control number of deathsprevented by shortcourse chemotherapy in China," International Journal of Epidemiology, vol. 29, no. 3, pp. 558-564, 2000.

[5] L. Gammaitoni and M. C. Nucci, "Using a mathematical model to evaluate the efficacy of TB control measures," Emerging Infectious Diseases, vol. 3, no. 3, pp. 335-342, 1997.

[6] C. J. Murray and J. A. Salomon, Using Mathematical Models To Evaluate Global Tuberculosis Control Strategies-A Paper Presented at the Centre For Population and Development Studies, Harvard University, Cambridge, Mass, USA, 1998.

[7] World health Organization, WHO: Tuberculosis Fact Sheet, 2007.

[8] O. K. Koriko and T. T. Yusuf, "Mathematical model to simulate tuberculosis disease population dynamics," American Journal of Applied Sciences, vol. 5, no. 4, pp. 301-306, 2008.

[9] D. Baleanu, K. Diethelm, E. Scalas, and J. J. Trujillo, Fractional Calculus Models and Numerical Methods Series on Complexity, Nonlinearity and Chaos, World Scientific, 2012.

[10] A. Atangana, A. Ahmed, and N. Belic, "A generalized version of a low velocity impact between a rigid sphere and a transversely isotropic strain-hardening plate supported by a rigid substrate using the concept of non-integer derivatives," Abstract Applied Analysis, vol. 2013, Article ID 671321, 9 pages, 2013.

[11] A. Atangana and J. F. Botha, "Analytical solution of the groundwater flow equation obtained via homotopy decomposition method," Journal of Earth Science \& Climatic Change, vol. 3, p. 115, 2012.

[12] A. Atangana and E. Alabaraoye, "Solving a system of fractional partial differential equations arising in the model of HIV infection of $\mathrm{CD}^{+}$cells and attractor one-dimensional KellerSegel equations," Advances in Difference Equations, vol. 2013, p. 94, 2013.

[13] A. Atangana and A. Secer, "Time-fractional coupled- the korteweg-de vries equations," Abstract Applied Analysis, vol. 2013, Article ID 947986, 8 pages, 2013.

[14] K. B. Oldham and J. Spanier, The Fractional Calculus, Academic Press, New York, NY, USA, 1974.

[15] I. Podlubny, Fractional Differential Equations, Academic Press, New York, NY, USA, 1999.

[16] A. A. Kilbas, H. M. Srivastava, and J. J. Trujillo, Theory and Applications of Fractional Differential Equations, Elsevier Science B.V., Amsterdam, The Netherlands, 2006.

[17] A. Atangana and A. Secer, "A note on fractional order derivatives and Table of fractional derivative of some specials functions," Abstract Applied Analysis, vol. 2013, Article ID 279681, 2013.

[18] M. Caputo, "Linear models of dissipation whose Q is almost frequency independent, part II," Geophysical Journal International, vol. 13, no. 5, pp. 529-539, 1967.

[19] S. G. Samko, A. A. Kilbas, and O. I. Marichev, Fractional Integrals and Derivatives: Theory and Applications, Gordon and Breach, Yverdon, Switzerland, 1993.

[20] D. Baleanu, K. Diethelm, E. Scalas, and J. J. Trujillo, Fractional Calculus, Series on Complexity, Nonlinearity and Chaos, World Scientific, 2012, Models and numerical methods.

[21] A. Atangana and A. Kılıçman, "A possible generalization of acoustic wave equation using the concept of perturbed derivative order," Mathematical Problems in Engineering, vol. 2013, Article ID 696597, 6 pages, 2013. 


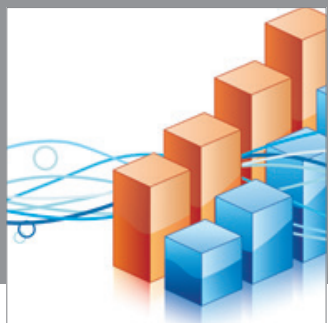

Advances in

Operations Research

mansans

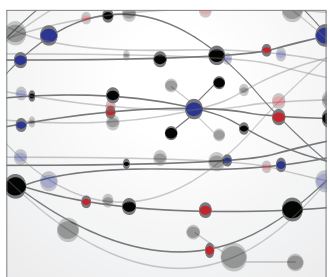

The Scientific World Journal
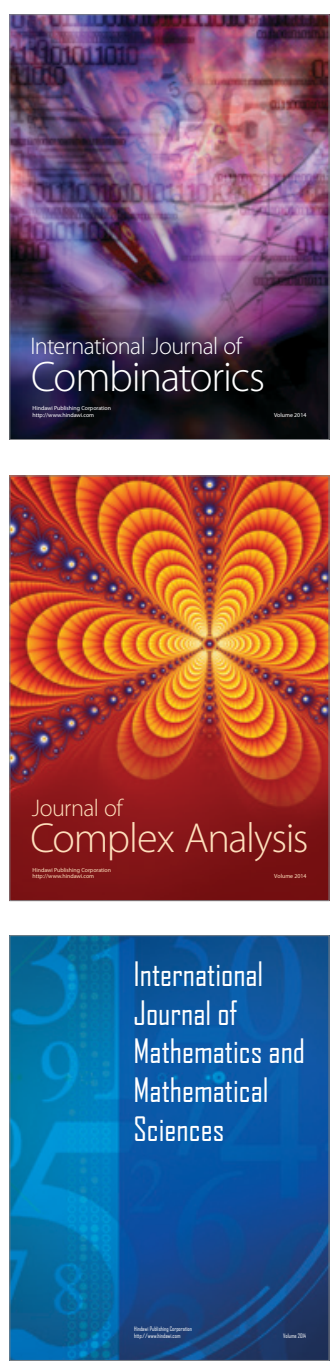
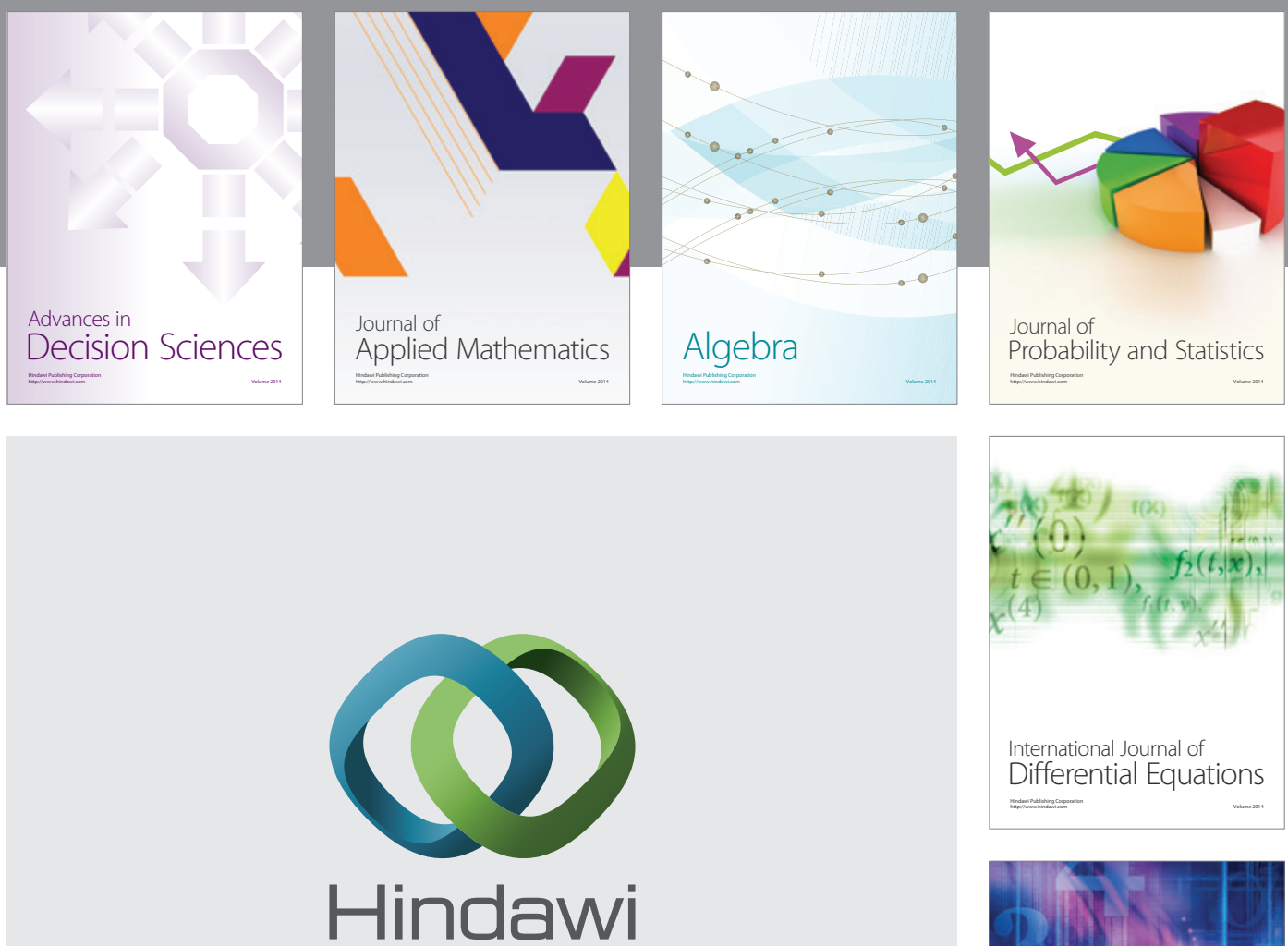

Submit your manuscripts at http://www.hindawi.com
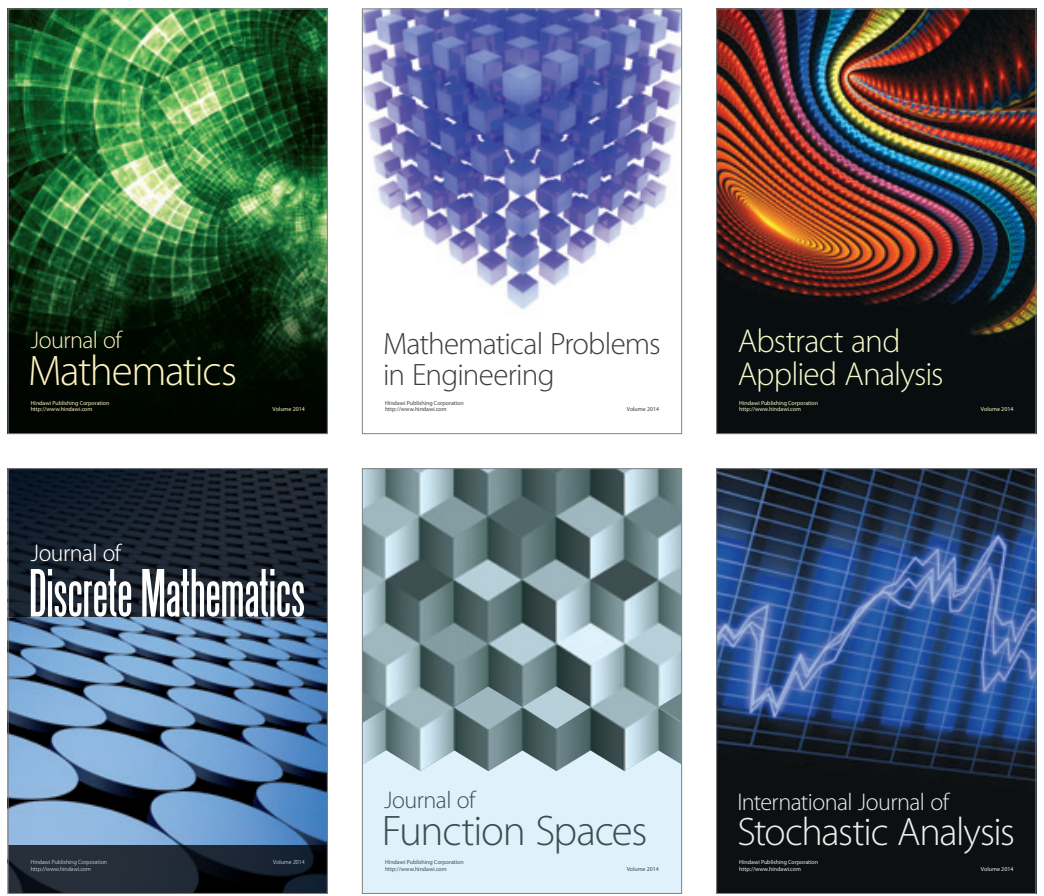

Journal of

Function Spaces

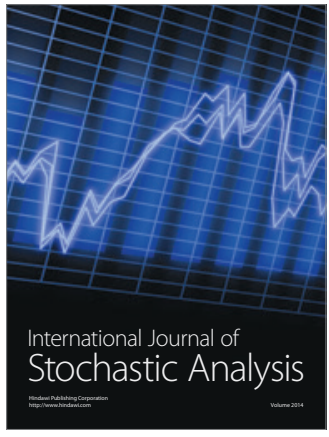

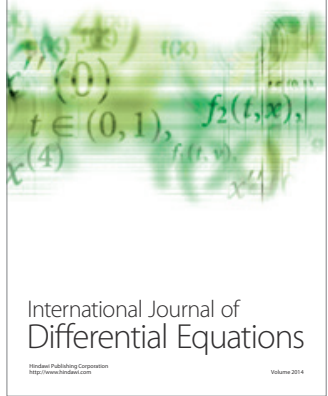
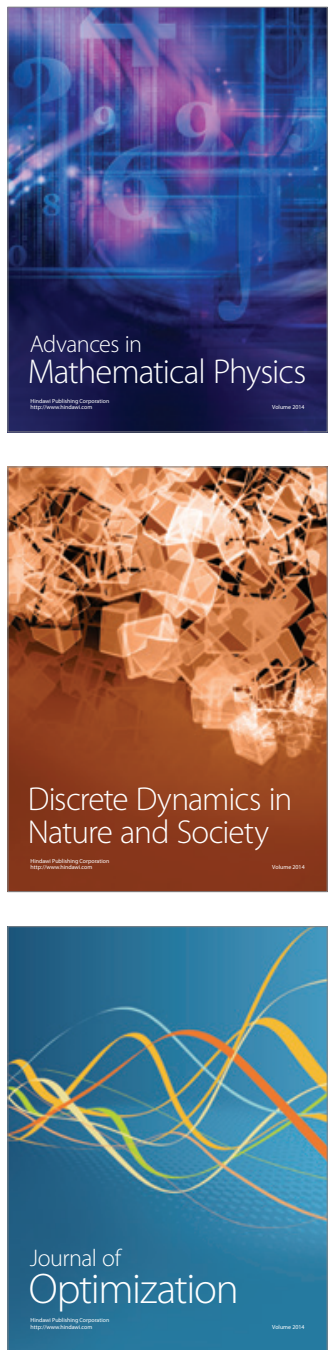